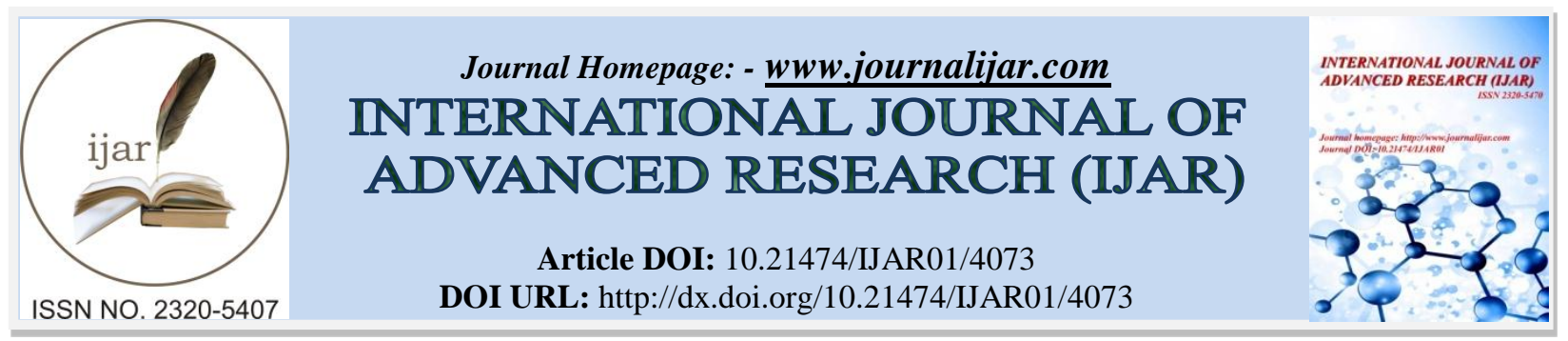

RESEARCH ARTICLE

\title{
NURSING PRACTICES AT THE BUEA REGIONAL HOSPITAL THAT PREDISPOSE CESAREAN SECTION PATIENTS TO POST-OPERATIVE COMPLICATIONS
}

\author{
"Samuel Nambile Cumber ${ }^{1,2}$, Njodzeka Bernard Wirndzem ${ }^{3}$, Kanjo Mireille Nuhow ${ }^{2,3}$, Ngunde Palle John ${ }^{3}$ \\ and Rosaline Yumumkah Kanjo Cumber ${ }^{2,4}$. \\ 1. Discipline of Public Health Medicine, Department of Nursing \& Public Health, College of Health Sciences, \\ University of Kwazulu-Natal, Durban, South Africa. \\ 2. Under Privileged Children and Women Assistance (UPCAWA-SWEDEN), Buea, Cameroon. \\ 3. Department of Nursing Sciences, University of Buea, Cameroon. \\ 4. Department of Political Science, University of KwaZulu-Natal, Durban, South Africa.
}

\section{Manuscript Info}

(...........................

Manuscript History

Received: 01 March 2017

Final Accepted: 41 April 2017

Published: May 2017

Key words:-

Nursing; Practice; Cesarean; Patient;

Complications

\section{Abstract}

This study was limited to nurses working in the maternity and theatre and to hospitalized postoperative cesarean section patients in the Buea Regional Hospital. Incidental sampling method was used. The entire cohort was targeted as well as medical past records concerning birth from January to May 2014. A questionnaire and check list was used to collect data. The study involved 24 nurses, 10 post-operative cesarean section patients and 453 past medical records. Data was processed using SPSS 17.0, Microsoft word and excel 2010. Data was presented using percentages, tables and charts. The rate of cesarean section in the BRH is $20.3 \%$. Post-operative complication was dominated by $8.7 \%$ for surgical site infection, $3.3 \%$ each for hemorrhage, respiratory difficulties and bladder puncture. $13.04 \%$ and $6.5 \%$ of the patients postoperatively developed severe epigastric pain and an offensive lochia respectively. The results showed that "nurses at the BRH have lapses in their nursing care practices that predispose cesarean section patients to post-operative complications". Seminars should be organized on the management of pre- and post-operative cesarean section patients.

Copy Right, IJAR, 2017,. All rights reserved.

\section{Introduction:-}

A cesarean section (CS) is a surgical operation used to extract one or more babies through an incision in the abdominal wall and the uterus American College of Obstetricians and Gynecologists (ACOG). ${ }^{[1]}$ Caesarean section may sometimes be the only means to save the life of the mother and/or fetus. ${ }^{[1,2]}$ The nurse' responsibility as a care giver in the prevention of cesarean complication is a legal liability the nurse owes to the woman, family members and team members. $\left.{ }^{[1,3}\right]$ By accepting responsibility in performing a task, the nurse must ensure the task is performed competently with the standards of an ordinary competent practitioner in the type of task such as basic management of surgical patients. ${ }^{[2,3]}$ If the nurse should delegate a task, such as inserting a urinary catheter, the nurse must be sure the person (nursing student, nursing assistant) understands the task (how it is performed), and has the surgical skills and ability in performing the task competently so as to prevent cesarean complications from occurring.

Corresponding Author:- Dr. Samuel Nambile Cumber. 
In Cameroon, a study in the Far-North Region reported hemorrhage and infection to be the main cesarean complications, with other complications such bladder injuries and hemorrhage to be the commonest complications. [4]

Coalition for Improving Maternity Services, is concerned with the dramatic increase and ongoing over use of CS. ${ }^{[4,5]}$ The Reproductive Care Program Nova Scotia published that the rates of CS increased from $6 \%$ in 1970 and $26 \%$ in 2006 Worldwide. ${ }^{[6,7]}$ The estimates in Cameroon put the national caesarean section rate at about 2 to $3 \%$. ${ }^{[7,8]} \mathrm{A}$ CS rate of $7 \%$ to $12 \%$ was reported at the University Teaching Hospital, Yaoundé. ${ }^{[8]}$

Some of the factors leading to changes in the CS rates include: clinical practice guidelines, attitudes and beliefs about birth, choice of the childbearing women and maternal demography. ${ }^{[6,8]}$ The surgical procedure poses short and ${ }_{[6-8]}$ long-term health risks to mother and infant. ${ }^{[1,2,8]}$ A scared uterus poses risk to all future pregnancies and deliveries.

Cesarean section accounts for $26.14 \%$ of surgeries performed in Cameroon. Surgical site infection (SSI) has a prevalence rate of $9.16 \%$. 2.87\% of these SSI occurs from CS which can be explained by poor prevention strategies of nosocomial infection. ${ }^{[5]}$ Hemorrhage, infection and bladder puncture has been reported to be the main CS complication. ${ }^{[4]}$

With all these, the researcher aims at finding out nursing care practices at the Buea Regional Hospital that predispose CS women to complications in the postoperative period. There are a lot of lapses in the pre- and postoperative management of women undergoing cesarean section by nurses, there by exposing these women to risk of developing cesarean complications after surgery?.

The objective was to explore the nursing care practices of nurses at the Buea Regional that predisposes cesarean section patients to post-operative complications.

Research Methodology:-

Area of Study and Setting: This study was carried out in the Buea Regional Hospital located in Fako Division, South West Region of the Republic of Cameroon.

\section{Study Design:-}

The study design was a descriptive, cross sectional prospective and retrospective study analyzed through quantitative method.

\section{Inclusion Criteria and Exclusion Criteria:-}

\section{Inclusion Criteria:-}

- Only nurses working in the maternity and theatre unit in the BRH.

- Only women who gave birth via CS, and Only medical records of women that gave birth between January and May 2014 were studied.

Exclusion Criteria:-

- $\quad$ Nurse working in other units except the theatre and maternity unit.

- Women who had given birth through the normal vagina delivery.

\section{Study Population and Sampling:-}

\section{The Study Population:-}

The study population was made up of nurses of the theatre and maternity units and patients who gave birth through cesarean section in the BRH. Also, all past medical records of patients who had given birth between the months of January to May, 2014 were studied.

\section{Sampling Technique Sample Size:-}

Sampling was by purposeful sampling where any nurse and patients who met the inclusion criteria were approached and asked to participate in the study. The entire cohort was targeted and over the study period, the sample size was 
made up of 24 nurses, 10 patients and 453 past medical records that was incidentally meet. All medical records within January to May, 2014 were studied.

\section{Study Procedures:-}

\section{Instrument for data collection:-}

A semi-structured questionnaire comprising close ended and open ended questions and a check list was used to collect data from nurses and post-operative CS patient respectively, and medical records in the maternity.

\section{Ethical consideration:-}

The instruments used in collecting data from both the nurses and patients were a questionnaire and check list. Also, past medical records were viewed to get information on rates of CS, CS complication and the different type of complications.

Written or verbal consent was gotten from the nurses and patients after self-presentation. The nurses and patients were briefed on the purpose of the research. The anonymity and confidentiality of the questionnaires were ensured. An authorization to carry out the research was gotten from the Faculty of Health Sciences through the Head of department and in the Buea Regional Hospital permission to carryout research was obtained from the Hospital Management through the Director of the BRH.

\section{Data Management and Analysis:-}

Open-ended questions was analysed using the process of thematic content analysis where by concepts or ideas were grouped under umbrella terms or key words to appreciate the trend of ideas or appreciations. Data was processed and analysed using SPSS 17.0 (SPSS Inc, 2008), Microsoft Excel 2010 The questionnaire was made essentially of categorical variables that were analysed using frequencies and proportions. Data was presented using frequency table and chart.

\section{Duration of Data Collection:-}

Data was collected from the $6^{\text {th }}$ to the $14^{\text {th }}$ of June, 2014 from nurses, post-operative CS patient, and past medical records.

\section{Results:-}

\section{Demography:-}

Age: Number of nurses studied $=24$, number of patients $=10$, and number of medical records reviewed $=453$ and number of $\mathrm{CS}=92$.

Table 1: Age Distribution of nurses in the BRH

\begin{tabular}{|c|c|c|c|c|}
\hline & Age & Frequency & Percent & Valid Percent \\
\hline \multirow[t]{6}{*}{ Valid } & $21-25$ & 3 & 12.5 & 14.3 \\
\hline & $26-30$ & 6 & 25 & 28.6 \\
\hline & $31-35$ & 7 & 29.2 & 33.3 \\
\hline & $36-40$ & 4 & 16.7 & 19 \\
\hline & $>40$ & 1 & 4.2 & 4.8 \\
\hline & Total & 21 & 87.5 & 100 \\
\hline \multicolumn{2}{|c|}{ No answer } & 3 & 12.5 & \\
\hline Total & & 24 & 100 & 100 \\
\hline
\end{tabular}

\section{Educational Qualification:-}

Five categories of nurses were studied: nurse aid, Higher Professional Diploma (HPD), Higher National Diploma (HND), Brevete nurses and State Registered Nurses (SRN nurses). Out of the 24 nurses, 7(31.8\%) were NA, 7(38.1\%) were SRN, 6(27.3\%) were Brevete nurses, 1 (4.5\%) each for Higher National Diploma (HND) and Higher Professional Diploma (HPD). The modal category was NA and SRN. 


\section{Pre and Post-Operative Nursing Management Practices of CS Patients in the BRH:- 4 Most Frequent Indications for CS.}

According to the result findings, Only 4(16.7\%) of nurses in the BRH knows the most frequent indications of cesarean section. The remaining 20(83.3\%) of the nurses do not know all the most frequent indications of CS.

Out of the 24 nurses studied, 21(87.5\%) had knowledge on CS complications and 14(58.3\%) could rightly determine more than 3 of the risk factor deep venous thrombosis (DVT). These risk factors included: age, immobility, sepsis, trauma, inflammation, obesity and pregnancy. This is illustrated on figure 1.

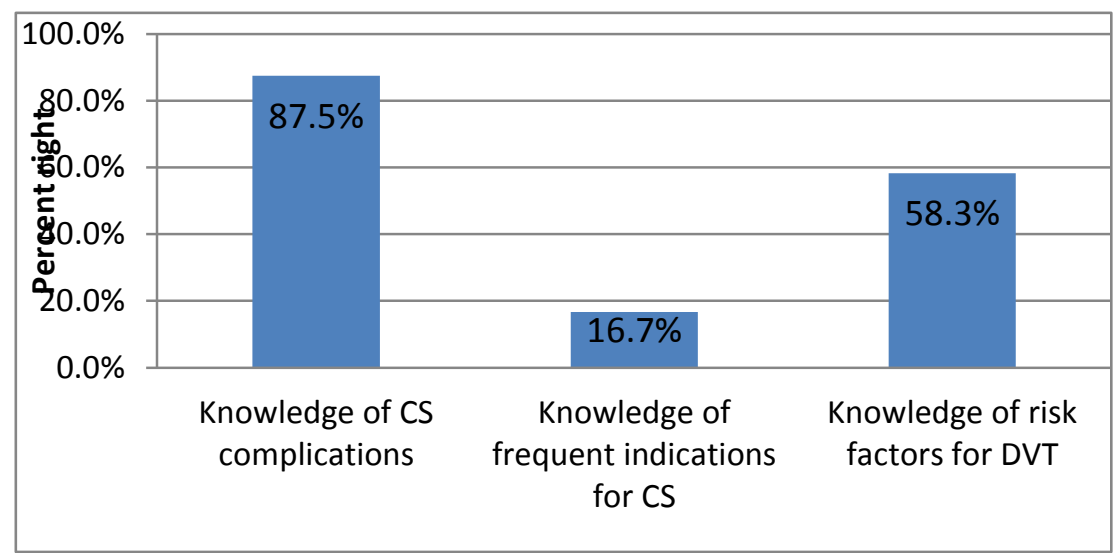

Figure 1:- Knowledge on CS

$9(37.5 \%)$ of the nurses confirmed that airway, breathing and circulation (ABC) must be immediately assessed postoperatively while the remaining $15(62.5 \%)$ of the nurse assessed other parameters other than "ABC" immediate post-OP.

The above results have been summarized in figure 2 below.

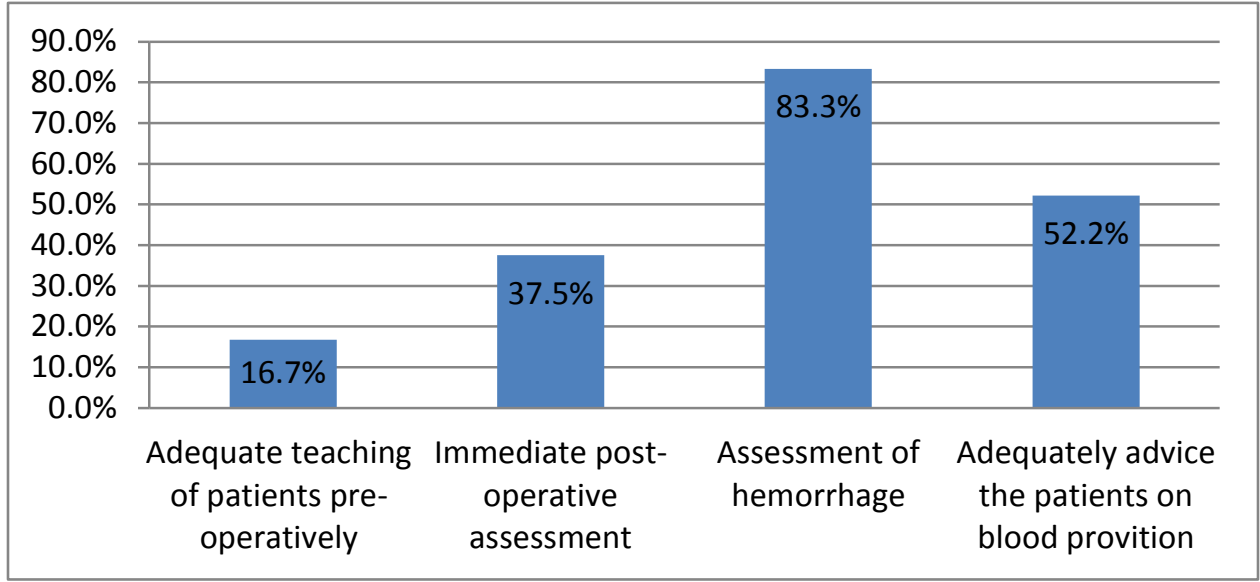

To Identify the Indications, Rate of CS, Rate of CS Complication, and the Various CS Complications that develops from Hospitalized CS patients at the BRH Maternity.

From January to June 2014, there have been a total of 453 births and 92 CS in the BRH. The rate of CS in the BRH, is ranged between $16.7 \%$ to $26.7 \%$ with an average CS rate of $20.3 \%$. The rates of CS from January, February, March, April and May are 15(18.5\%), 15(20.8\%), 18(16.7\%), 24(26.7\%), and 20(19.6\%) respectively. This is illustrated by table 2 bellow.

The 4 most frequent indications of CS in the BRH are: 21(22.8\%) for Repeat CS, 15(16.3\%) for arrest of labour, $12(13.0 \%$ formal-presentation and 11(12.0\%) for fetal distress. These 4 factors account for $68.1 \%$ of CS in the BRH. Indicators for CS section are summarized in table 2 bellow. 
Table 2:- Demonstrates the different Indicators for CS in the BRH

\begin{tabular}{|l|l|l|l|}
\hline SN & Indication for cesarean section in the BRH & Frequency & Weight (\%) \\
\hline 1 & Repeat Cesarean section & 21 & $22.8 \%$ \\
\hline 2 & arrest of labour & 15 & $16.3 \%$ \\
\hline 3 & Malpresentation & 12 & $13.0 \%$ \\
\hline 4 & Fetal distress & 11 & $12.0 \%$ \\
\hline 5 & Macrosomial baby & 4 & $4.3 \%$ \\
\hline 6 & pre-eclampsia/eclampsia & 5 & $5.4 \%$ \\
\hline 7 & Multiple pregnancy & 3 & $3.3 \%$ \\
\hline 8 & placenta previa & 4 & $4.3 \%$ \\
\hline 9 & Cephalopelvic Disproportion & 5 & $5.4 \%$ \\
\hline 10 & Elderly Primi & 4 & $4.3 \%$ \\
\hline 11 & Others & 8 & $8.7 \%$ \\
\hline 12 & Total & 92 & $100.0 \%$ \\
\hline
\end{tabular}

From the months of January to May 2014, there were 92 CS and 23 of the CS developed complication. The rate of post-operative CS complication in the BRH is $25.0 \%$. 8(8.7\%) of the patients developed surgical site infection, hemorrhage and bladder puncture was developed in 3(3.3\%) of the patients each. 3(3.3\%) of the patients had respiratory difficulties postoperatively. $2(2.2 \%)$ of the patients each developed endometritis and urinary tract infection (UTI). Out of the 92 medical records of CS patients that where studied, 12(13.04\%) of the patients postoperatively developed severe epigastric pain. $33(35.9 \%)$ developed fever $\left(38.5^{\circ} \mathrm{C}\right) .14(15.2 \%)$ of the women were postoperatively transfused and $6(6.5 \%)$ of the women developed an offensive lochia. Post-OP CS complications are illustrated on figure 3 bellow.

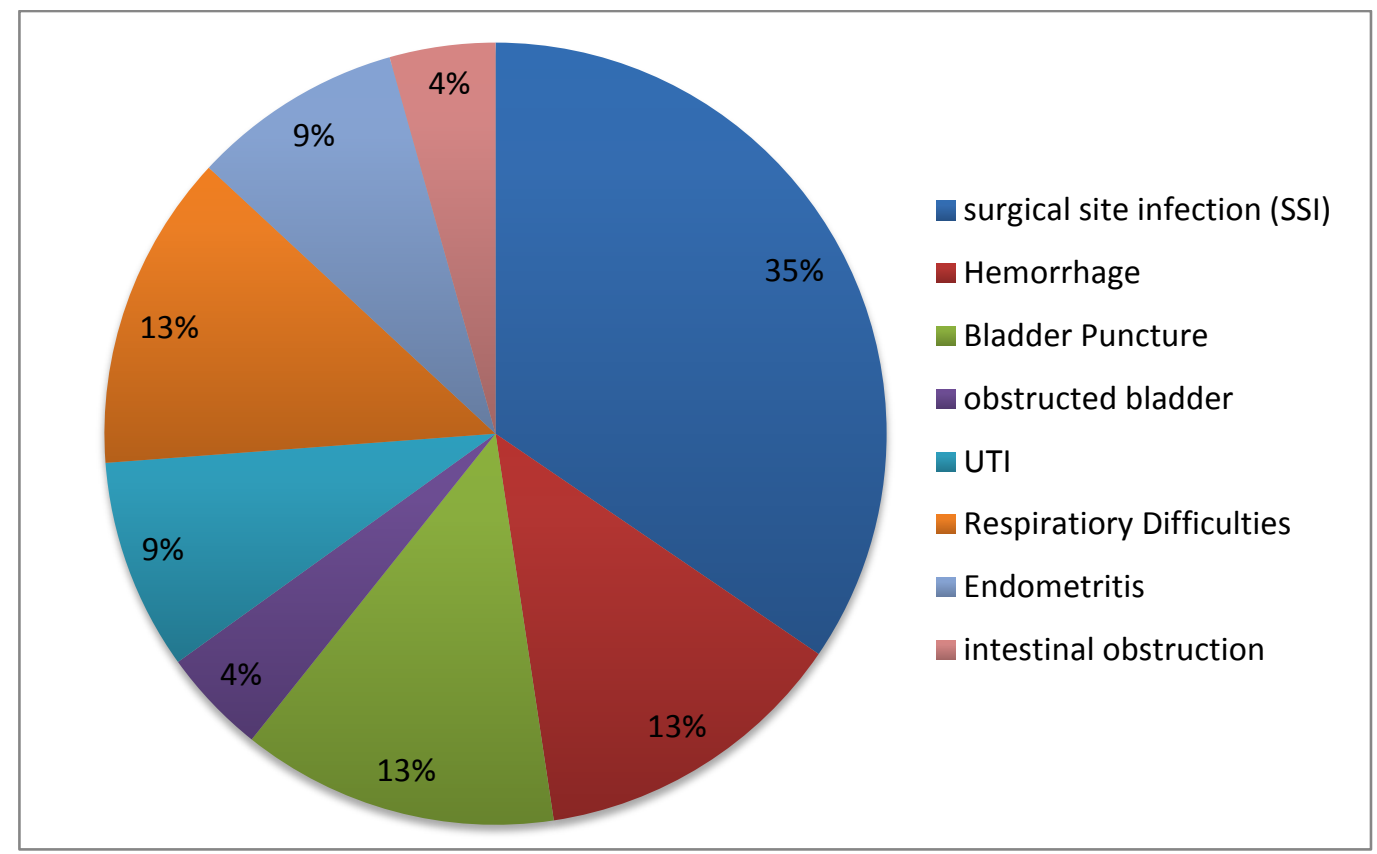

Figure 3:- Post-OP CS complications in the BRH.

\section{Nursing Care Practice at the BRH Predisposing Patients to post CS Complication:-}

Out of the 24 nurses studied, 19(79.2\%) of the nurses said they adequately monitor vital signs post-OP while the remain $5(20.8 \%)$ of the nurses said they find it difficult to follow the protocol for monitoring Vital signs post-OP. $22(91.7 \%)$ of the nurses said they immediately replaced soaked dressing while $2(8.3 \%)$ of the nurses said they apply additional dressing to existing ones. 


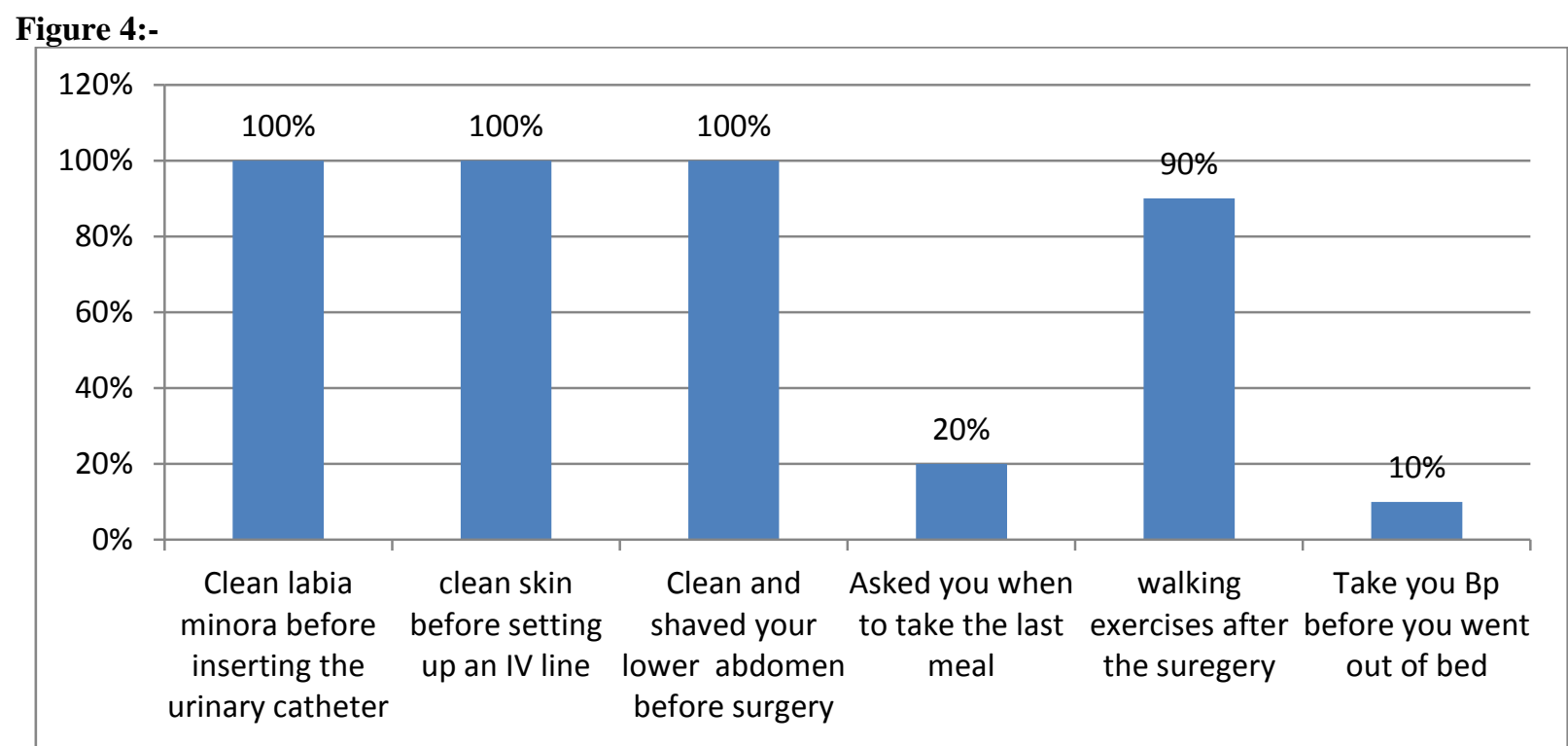

Table 3:- CS Problem faced by post-operative patients

\begin{tabular}{|l|l|l|l|}
\hline Patient problem & Number $(\mathbf{n})$ & $\mathbf{N}$ & Percentage (\%) \\
\hline Delayed healing & 1 & 10 & 10 \\
\hline Fever & 3 & 10 & 30 \\
\hline Severe pain & 2 & 10 & 20 \\
\hline Infection & 2 & 10 & 20 \\
\hline Urinary obstruction & 1 & 10 & 10 \\
\hline Epigastric pain & 3 & 10 & 30 \\
\hline
\end{tabular}

\section{Discussion:-}

Pre and Post-Operative Management Practices of Cesarean Section patients:-

Only 4(16.7\%) of nurses in the Buea Regional Hospital had knowledge on the 4 most frequent indication of CS in the world. The 4 most frequent indications of CS in the BRH were; repeat CS $=21(22.8 \%)$, arrest of labour $=$ $15(16.3 \%)$, mal-presentation $=12(13.0 \%)$ and fetal distress $=11(12.0 \%)$. Bulger et al., (1998) said these 4 indicators account for $67 \%$ of all CS which is similar to $68.1 \%$ of CS in the BRH with a difference of $1.1 \%$. $21(87.5 \%)$ of the nurses had a good knowledge on CS complication so the nurses could better prevent complications from occurring. 14(58.3\%) of the nurses could identify most of the risk factors of deep venous thrombosis (DVT) but little was done in preventing DVT. 9(37.5\%) of the nurses immediately assess airway, breathing and circulation $(\mathrm{ABC})$. Post-operative patients are at risk of developing severe complications such as hypoxia and hypotension which might lead to brain damage and shock respectively. The above results show similarities with publications from Robert Dyson, Suzzanne, C. S, Branda, G. ${ }^{[9,10]}$

\section{Complication that Develop from Cesarean Section among Hospitalized CS Patients:-}

Out of the 453 births between Januarys to May 2014, there were 92 CS. The rate of CS in the BRS is between $18(16.7 \%)$ to $24(26.7 \%)$. The average rate of CS in the BRH is $20.3 \%$. This is far more than the national CS rate of 2 to $3 \%$ and a rate of $7-12 \%$ at the University Teaching Hospital, Yaoundé by Nana et al. ${ }^{[4]}$ The difference in CS rate in the BRH compared to rates in CHU is $10.8 \%$. This is because the BRH manages mostly referred patients indicated for CS in the in the Buea health district. 
According to a study conducted by Dipanda in Yaoundé Cameroon, the types of CS complication included $1.9 \%$ for hemorrhage and $2.3 \%$ for bladder injuries. ${ }^{[11]} \mathrm{SSI}=8.7 \%$ is the most common complication in the BRH which is greater, as reported in another study by Ntsama et al, that was conducted in Yaoundé in 3 public hospitals revealed that the rate of CS SSI is $2.87 \%$. ${ }^{[5]}$ This is followed by hemorrhage, bladder puncture and respiratory difficulties is similar to the studies conducted by Nana and Dipanda. ${ }^{[4,11]} 12(13.04 \%)$ of the patients developed severe epigastric pain post-operatively which is a sing of starvation which indirectly affects wound healing as the protein used in wound healing is instead converted as energy. Two reports from ACOG are of the opinion that fluids can be allowed soon after surgery and light diet may be started when the woman feels ready to eat. ${ }^{[1,2]}$ Initiation of oral maternal feeding following Cs is safe and beneficial to the recovery of the mother. ${ }^{[3,6]} 33(35.9 \%)$ of the patients developed fever $\left(38.5^{\circ} \mathrm{C}\right)$ which is a sign of systemic infection.

\section{Nursing Care Practices Predisposing CS Patients to Post-Operative Complications:-}

Only $6(27.3 \%)$ of the nurses said they prevented respiratory tract infection by placing the patient's head on the side to prevent aspiration of gastric content and also to encourage airway clearance. None of the nursed taught patient deep breathing and coughing exercises to clear off and mobilize secretion in the respiratory tract. This clearly indicates that post-operative CS patients are at high risk of developing RTI. None of the nurses mentioned hand washing as an effective way of preventing infection. Patients in the BRH are at risk of developing nosocomial infections as in Ntsama. ${ }^{[5]}$ A very important aspect of nursing practice is left out during the management of CS patients which is bed making, and this has also been mention by Babara in her report. ${ }^{[6]}$ During bed making, a milieu for effective education, better nurse-patient relationship and assessment of the surgical site and its immediate environment is created as similarities was reported by Suzanne et al. ${ }^{[10]} 10(41.7 \%)$ of the nurses could effectively manage DVT by ambulating and doing leg exercise with the patients. This could be because the nurses have inadequate knowledge and skills in assessing and preventing DVT. Nurses will better manage a complication if they can easily identify risk factors to it. ${ }^{[6,7]}$

\section{Conclusion:-}

Most of the nurses in the BRH have average skills on managing women indicated for surgery pre- and postoperatively. Some aspects of nursing is just left out. To conclude, nurses at the BRH have lapses in their nursing care practices that predispose CS patients to post-operative complications.

\section{Recommendations:-}

In order to prevent nursing care practices predisposing cesarean section patients to post-operative complications at the Buea Regional Hospital, the study recommends

- Seminars should be organized on pre- and post-operative management of cesarean section patients, early identification of complications and how to CS complications.

- Standardized protocols on how to manage patients pre- and post-operatively should be introduced into our health institutions for better management of cesarean sections.

- A recovery room should be created in all institutions carrying out cesarean sections for better post-operative management of the patients and immediate resuscitation in case of immediate complications.

- A data base should be created on cesarean section to evaluate changes in practice with time.

\section{References:-}

1. American College of Obstetricians and Gynecologists (ACOG). (2010). Antimicrobial prophylaxis for cesarean delivery - timing of administration. Retrieved $4^{\text {th }}$ April, 2014 from http://www.acog.org/Resources\% 20.

2. ACOG. (2011). FAQ labor, delivery, and postpartum care: cesarean birth. Retrieved $4^{\text {th }}$ April, 2014 from http://www.acog.org/ /media/For\%20Patients/pdf

3. Serivener R. et al. (2011). Accountability and Responsibility (vol 25). RCN publishing Company.

4. Nana, P. N., Fomulu, J. N., Djenabow, A., Mbu, R., Tonye, R., V'andji, J., Leke, R. (2011). Epidemio-Clinical Factors Associated with Cesarean Section in Two Referral Hospital. Far North Region, Cameroon. Ashdin Publishing Clinical in Mother and Child Health. p. 5.

5. Ntsama Essomba, Avomo, J., Esiene, A., Leme, B., Abolongo, A., Masso, M., Essomba, M. (2013). Prevalence of SSI and evaluation of risk factors after surgery. Journal of Medicine and Dedical Science, 4(6), 241-246. http//interesjournal.org.

6. Barbara, R. H., Barbara, A., Esther, C. (2010). Nursing Assessment and a Nursing Process Approach Basic. Clifton Park: Delmar. 
7. Althabe, F., Sosa, C., Belizán, J. M., Gibbons, L., Jacquerioz, F., Bergel, E. (2006). Cesarean section rates and maternal and neonatal mortality in low-, medium-, and high-income countries: an ecological study. Birth, 33(4):270-277

8. Tebeu, P. M., Ngassa, P., Mboudou, E., et al. (2008). Neonatal survival following cesarean delivery in northern Cameroon. International Journal of Gynecology and Obstetric, 103(3), 259-260.

9. Robert Dyson. (2012). Cesarean Delivery: Surgical Techniques. ISBN: 978-953. Available from Http://www.intechopen.comlbookslc.delivery.

10. Suzzanne, C. S, Branda, G., Janice, L., Kerry, H. (2010). Brunner and Suddarth's Test Book of Medico-Surgical Nursing (12 $2^{\text {th }}$ ed.). Philadelphia: Lippicott Williams.

11. Dipanda T.D. (1995). Les complications maternelles des $\mathrm{c}^{\prime}$ esariennes dans nos $\mathrm{h}^{\wedge}$ opitaux. MD thesis, Faculty of Medicine and Biomedical Sciences. Yaoundé, Cameroon: University of Yaoundé I. 